\title{
HIGH-PRESSURE STUDY OF TI50Ni25Fe25 POWDER PRODUCED BY MECHANICAL ALLOYING
}

\author{
Ferreira, A. S. ${ }^{1 *}$; Rovani, P. R. ${ }^{2}$; de Lima, J. C. ${ }^{3}$; Pereira, A. S. ${ }^{4}$ \\ ${ }^{1}$ Departamento de Engenharia Mecânica, Universidade Federal de Santa Catarina, Florianópolis, Santa Catarina, Brazil \\ ${ }^{2}$ Instituto de Física, Universidade Federal do Rio Grande do Sul, Porto Alegre, Rio Grande do Sul, Brazil \\ ${ }^{3}$ Departamento de Física, Universidade Federal de Santa Catarina, Florianópolis, Santa Catarina, Brazil \\ ${ }^{4}$ Departamento de Materiais, Escola de Engenharia, Universidade Federal do Rio Grande do Sul, Porto Alegre, Rio Grande do Sul, Brazil
}

\section{*ailtonsfn@hotmail.com}

A nanostructured $\mathrm{Ti}_{50} \mathrm{Ni}_{25} \mathrm{Fe}_{25}$ phase (B2) was formed by mechanical alloying and its structural stability was studied as a function of pressure. The changes were followed by X-ray diffraction. The B2 phase was observed up to $7 \mathrm{GPa}$, and for larger pressures, the B2 phase transformed into a trigonal/hexagonal phase (B19) that was observed up to the highest pressure used (18 GPa). Besides B2 and B19, a segregation of elemental Ni and the formation of $\mathrm{FeNi}_{3}$ were observed. With decompression, the B2 phase was recovered. Values of bulk modulus for the B2, B19, elemental $\mathrm{Ni}$ and $\mathrm{FeNi}_{3}$ phases were obtained by fitting the pressure dependence of the volume to a Birch-Murnaghan equation of state (BMEOS). 